Pacific Journal of Mathematic 


\title{
GROUPS OF MATRICES ACTING ON DISTRIBUTION SPACES
}

\author{
S. R. HARASYMIV
}

\begin{abstract}
Let $E$ be a locally convex space of temperate distributions on the $n$-dimensional Euclidean space $R^{n}$, and $G$ aclosed subgroup of $\mathrm{Gl}(n, R)$, the general linear group over $R^{n}$. An attempt is made to identify those distributions which can be approximated in $E$ by linear combinations of distributions of the form $u(A x+b)$, where $u$ is a fixed element of $E, A$ varies over $G$, and $b$ varies over $R^{n}$. A cancellation theorem is proved; this then allows the support of the Fourier transform of any annihilator of the set of distributions of the form $u(A x+b)$ to be localized. This in turn is used to obtain approximation results.
\end{abstract}

1. Notation. Throughout, $R^{n}$ denotes $n$-dimensional Euclidean space. The character group of $R^{n}$ is again $R^{n}$, the identification being made in such a way that multiplicative factors in the Fourier inversion formula are eliminated. The Haar measure on $R^{n}$ is denoted by $d x$.

We denote by $C_{c}^{\infty}(U)$ the space of indefinitely differentiable functions on $R^{n}$ which have compact support inside the open set $U$ in $R^{n}$. $S\left(R^{n}\right)$ is the space of rapidly decreasing indefinitely differentiable functions on $R^{n}$. The space of all Schwartz distributions on $R^{n}$ is designated by $D^{\prime}\left(R^{n}\right)$, and its subspace consisting of temperate distributions is denoted by $S^{\prime}\left(R^{n}\right)$.

Gl $(n, R)$ is the general linear group over $R^{n}$. The determinant of an element $A$ in $\mathrm{Gl}(n, R)$ is written $\operatorname{det} A$, and $A^{\prime}$ denotes the adjoint matrix of $A$.

Now, consider a fixed element $A$ in $\operatorname{Gl}(n, R)$. Then it is easy to see that the function

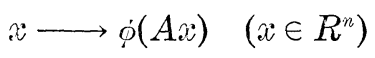

belongs to $C_{c}^{\infty}\left(R^{n}\right)$ whenever $\dot{\rho}$ does. We write $\dot{\phi}^{A}$ for this function. This definition is extended to include all distributions by making use of the adjoint of the map which carries $\dot{\phi}$ onto $\dot{\phi}^{A}$. More precisely, if $u$ is a distribution, then we define $u^{A}$ to be the unique distribution which satisfies

$$
\left\langle\dot{\phi}, u^{A}\right\rangle=\left|\operatorname{det} A^{-1}\right|\left\langle\phi^{A-1}, u\right\rangle \quad\left(\phi \in C_{c}^{\infty}\left(R^{n}\right)\right) .
$$

The translate of a distribution $u$ by an element $b$ in $R^{n}$ is defined in the usual fashion, and denoted by $u_{b}$. We write $u_{b}^{A}$ for $\left(u_{b}\right)^{A}$. 
These notational conventions entail that when $u$ is a function, then $u_{b}^{A}$ is the function defined by

$$
u_{b}^{A}(x)=u(A x+b) \quad\left(x \in R^{n}\right) .
$$

Let $G$ be a closed subgroup of $\operatorname{Gl}(n, R)$. We write $G^{\prime}$ for its adjoint group. $\boldsymbol{G}$ will always be equipped with the topology induced by $\operatorname{Gl}(n, R)$. With this topology, $G$ is a Lie group. We write $d A$ for left Haar measure on $\boldsymbol{G}$. If $\boldsymbol{V}$ is an open set in $G$, then $C_{c}^{\infty}(V)$ denotes the set of all smooth functions on $G$ having compact support in $V$.

Finally, again consider a closed subgroup $G$ of $\operatorname{Gl}(n, R)$. If $E$ is a locally convex space of temperate distributions which is invariant under all mappings of the form $u \rightarrow u^{A}$, where $A$ is a fixed element of $G$, we say that $E$ is $G$-invariant. Similarly, if $E$ is invariant under all maps of the form $u \rightarrow u_{b}$, where $b$ belongs to $R^{n}$, then we say that $E$ is translation invariant. Now, suppose that $E$ is both translation invariant and $G$-invariant, and that $u$ is an element of $E$. We denote by $T_{c}[u]$ the closed linear subspace of $E$ spanned by the set of all distributions of the form $u_{b}^{A}$, with $A$ in $G$ and $b$ in $R^{n}$.

2. Preliminaries. We shall be concerned with the problem of characterizing $T_{G}[u]$; that is, given two distributions $u$ and $w$ in $E$, when can we say that $w$ belongs to $T_{G}[u]$ ? Without some restrictions on $E$, the problem is a rather intractable one. However, quite mild assumptions about $E$ enable us to obtain a great deal of information about $T_{c}[u]$. These assumptions are mild in the sense that they allow us to include most of the classical distribution spaces in our results. In essence, the assumptions are that the topology on $E$ is barrelled, and that the action of the topological dual of $E$ can be expressed as a "convolution". We examine this situation below.

Definition 2.1. (cf. [1]). A locally convex space of temperate distributions is admissible if

(i) $S\left(R^{n}\right)$ is a dense subspace of $E$,

(ii) The inclusion maps $S\left(R^{n}\right) \rightarrow E \rightarrow S^{\prime}\left(R^{n}\right)$ are continuous.

The topological dual space of an admissible space can be identified with a subspace of $S^{\prime}\left(R^{n}\right)$ which contains $S\left(R^{n}\right)$. We shall always make this identification in such a way that the relations

$$
\begin{array}{ll}
\langle\dot{\phi}, v)=\dot{\phi} * v(0) & \left(\dot{\phi} \in S\left(R^{n}\right), v \in E^{\prime}\right) \\
\langle u, \phi\rangle=u * \dot{\phi}(0) & \left(u \in E, \phi \in S\left(R^{n}\right)\right)
\end{array}
$$

hold. 
Definition 2.2. Let $G$ be a closed subgroup of $\mathrm{Gl}(n, R)$. If $E$ is a $G$-invariant and translation invariant admissible space, we say that $E$ is a $G$-space if it has the following properties.

(i) For each $u$ in $E$, the map $x \rightarrow u_{x}$ is continuous from $R^{n}$ into $E$ for the weak topology on $E$.

(ii) For each $x$ in $R^{n}$, the map $u \rightarrow u_{x}$ is weakly continuous from $E$ into $E$.

(iii) For each $u$ in $E$, the map $A \rightarrow u^{A}$ is continuous from $G$ into $E$ for the weak topology on $E$.

(iv) For each $A$ in $G$, the map $u \rightarrow u^{A}$ is weakly continuous from $E$ into $E$.

(v) For each $u$ in $E$ and each $v$ in $E^{\prime}$, the continuous (by (i)) function $x \rightarrow\left\langle u_{x}, v\right\rangle$ is a temperate distribution on $R^{n}$.

We shall find it convenient to denote by $u * v$ the function in $(\mathrm{v})$ above.

The next result lists some of the basic properties of $G$-spaces. These properties will be used repeatedly and without further reference in what follows.

Proposition 2.3. Let $E$ be a $G$-space, where $G$ is a closed subgroup of $\mathrm{Gl}(n, R)$. Then

(a) $E^{\prime}$ is translation invariant, and for each $b$ in $R^{n}$

$$
u * v_{b}=u_{b} \bar{*} v=(u \bar{*} v)_{b} \quad\left(u \in E, v \in E^{\prime}\right),
$$

(b) $E^{\prime}$ is G-invariant, and for each $A$ in $G$

$$
u \approx v^{A}=\left|\operatorname{det} A^{-1}\right|\left(u^{A-1} \mp v\right)^{A} \quad\left(u \in E, v \in E^{\prime}\right) .
$$

If $E$ is barrelled, then we have also

(c) $E^{\prime}$ is a module over $S\left(R^{n}\right)$ with respect to convolution, and for each $\phi \in S\left(R^{n}\right)$

$$
u \bar{*}(v * \phi)=(u \bar{*} v) * \dot{\phi} \quad\left(u \in E, v \in E^{\prime}\right) .
$$

Properties (a) and (b) reflect the fact that the maps $u \rightarrow u_{b}$ and $u \rightarrow$ $u^{A}$ of $E$ into $E$, being weakly continuous, have adjoints which are weakly continuous from $E^{\prime}$ into $E^{\prime}$. Property (c) derives from the fact that, if $E$ is barrelled, the map $u \rightarrow u * v$ is continuous from $E$ into $S^{\prime}\left(R^{n}\right)$, for each fixed $v$ in $E^{\prime}$. This may be verified by making use of the Closed Graph theorem; see [2].

Most of the classical spaces of temperate distributions are Gl ( $n$, $R$ )-spaces, and hence $\boldsymbol{G}$-spaces for every closed subgroup $\boldsymbol{G}$ of Gl ( $n$, $R$ ). The following result identifies a large class of $G$-spaces. However, since we shall not need this result in what follows, we omit its proof. 
Proposition 2.4. Let $G$ be a closed subgroup of $\mathrm{Gl}(n, R)$ and $E$ a G-invariant and translation invariant admissible space. Suppose that $E$ is barrelled, $B_{r}$-complete and a module over $S\left(R^{n}\right)$ with respect to convolution. Then $E$ is a $G$-space if and only if the following conditions hold.

(i) For each $u$ in $E$, the map $x \rightarrow u_{x}$ of $R^{n}$ into $E$ is bounded on compact subsets of $R^{n}$.

(ii) For each $u$ in $E$, the map $A \rightarrow u^{A}$ of $G$ into $E$ is bounded on compact subsets of $\boldsymbol{G}$.

3. The function $q \nabla F$. In this section, we construct a certain function, which will serve as the basic tool in proving our approximation results. Before we carry out this construction, it is convenient to introduce some additional notation.

Suppose that $\boldsymbol{H}$ is a closed subgroup of $\mathrm{Gl}(n, R)$, of Lie group dimension $m \geqq n$. Let $\theta:\left(t_{1}, \cdots, t_{m}\right) \rightarrow \theta\left(t_{1}, \cdots, t_{m}\right)$ be a diffeomorphism of a neighborhood of the origin in $R^{n}$ onto a neighborhood of the identity in $\boldsymbol{H}$, and such that $\theta(0, \cdots, 0)$ is the identity matrix. We write $\theta_{j k}\left(t_{1}, \cdots, t_{m}\right)$ for the $(j, k)$ th matrix element of $\theta\left(t_{1}, \cdots, t_{m}\right)$. Let 1 be the set of all subsets of $\{1, \cdots, m\}$ which have cardinality $n$. Suppose that $i=\left\{i_{1}, \cdots, i_{n}\right\}$ is an element of $I$ (we adopt this notation because we shall be using $I$ as an index set). We define a polynomial $P_{i}$ on $R^{n}$ by setting $P_{i}(x)=P\left(x_{1}, \cdots, x_{n}\right)$ to be the determinant which has, as its $(j, k)$ th entry, the expression

$$
x_{1} \frac{\partial \theta_{j_{1}}}{\partial t_{i_{k}}}+x_{2} \frac{\partial \theta_{j 2}}{\partial t_{i_{k}}}+\cdots+x_{n} \frac{\partial \theta_{j n}}{\partial t_{i_{k}}}
$$

evaluated at $t_{1}=t_{2}=\cdots=t_{m}=0$. Then each $P_{i}$ is a homogeneous polynomial of degree $n$. We write $Z(\boldsymbol{H})$ for the set of common zeros of these polynomials; that is,

$$
Z(\boldsymbol{H})=\bigcap_{i \in I}\left\{x: P_{\imath}(x)=0\right\} .
$$

We can now turn our attention to the promised construction. Let $\boldsymbol{H}$ be a closed subgroup of $\mathrm{Gl}(n, R)$. Consider a distribution $q$ in $D^{\prime}\left(R^{n}\right)$ and a function $F$ in $C_{c}^{\infty}(\boldsymbol{H})$. If $\phi$ is in $C_{c}^{\infty}\left(R^{n}\right)$, then the mapping

$$
A \longrightarrow\left\langle q^{A}, \phi\right\rangle \quad(A \in G)
$$

defines a continuous function on $\boldsymbol{H}$. It then follows that we can define a linear functional

$$
\dot{\phi} \longrightarrow \int_{\boldsymbol{H}}\left\langle q^{A}, \dot{\phi}\right\rangle F(A) d A
$$


on $C_{c}^{\infty}\left(R^{n}\right)$. We claim that this functional is continuous, and hence a distribution on $R^{n}$. For if $\left(\phi_{i}\right)$ converges to $\phi$ in $C_{c}^{\infty}\left(R^{n}\right)$, then it does so uniformly on equicontinuous subsets of $D^{\prime}\left(R^{n}\right)$. Now, the set $\left\{q^{A}: A \in \operatorname{supp} F\right\}$ is the image in $D^{\prime}\left(R^{n}\right)$ of the compact subset supp $F$ of $\boldsymbol{H}$ under the map $A$ to $q^{A}$. Since this map is continuous for the weak topology on $D^{\prime}\left(R^{n}\right),\left\{q^{A}: A \in \operatorname{supp} F\right\}$ is weakly compact as a subset of $D^{\prime}\left(R^{n}\right)$. It is thus an equicontinuous subset of $D^{\prime}\left(R^{n}\right)$ (because $C_{c}^{\infty}\left(R^{n}\right)$ is barrelled) and so

$$
\lim _{i}\left\langle q^{A}, \phi_{i}\right\rangle=\left\langle q^{A}, \phi\right\rangle
$$

uniformly for $A$ in $\operatorname{supp} F$. Form this, it follows that

$$
\lim _{i} \int_{H}\left\langle q^{A}, \phi_{i}\right\rangle F(A) d A=\int_{H}\left\langle q^{A}, \phi\right\rangle F(A) d A
$$

which proves our assertion.

In view of the above, we may now define a distribution $q \nabla F$ on $R^{n}$ by setting

$$
\langle\phi, q \nabla F\rangle=\int_{H}\left\langle q^{A}, \phi\right\rangle F(A) d A \quad\left(\phi \in C_{c}^{\infty}\left(R^{n}\right)\right) .
$$

Using a similar argument, with $S\left(R^{n}\right)$ in place of $C_{c}^{\infty}\left(R^{n}\right)$, it can be shown that $q \nabla F$ is a temperate distribution whenever $q$ is.

We have the following result.

LEMma 3.1. Suppose that $\boldsymbol{H}$ has dimension $m \geqq n$. Then $q \nabla F$ is an indefinitely differentiable function on $R^{2} \backslash Z(\boldsymbol{H})$ for every $q$ in $D^{\prime}\left(R^{n}\right)$ and $F$ in $C_{c}^{\infty}(H)$. Moreover, for each multi-index $\alpha$

$$
D^{\alpha}(q \nabla F)=\sum_{\| \beta|\leqq| \alpha}\left(D^{\beta} q\right) \nabla\left(G_{\beta}^{\alpha} F\right)
$$

where each $G_{\beta}^{\alpha}$ is a smooth function on $\boldsymbol{H}$ depending only on $\alpha$ and $\beta$.

Proof. The proof proceeds in several stages.

First, we make the observation that if $f$ is a continuous function on $R^{n}$, then for each $\phi$ in $C_{c}^{\infty}\left(R^{n}\right)$, and each $F$ in $C_{c}^{\infty}(\boldsymbol{H})$

$$
\begin{aligned}
\langle\phi, f \nabla F\rangle & =\int_{H}\left\langle f^{A}, \phi\right\rangle F(A) d A \\
& =\int_{H}\left\{\int_{R^{n}} f(A x) \phi(-x) d x\right\} F(A) d A \\
& =\int_{R^{n}} \phi(-x)\left\{\int_{H} f(A x) F(A) d A\right\} d x
\end{aligned}
$$

from which it follows that $f \nabla F$ is the function 


$$
f \nabla F(x)=\int_{H} f(A x) F(A) d A .
$$

It is easy to see that this function is continuous on $R^{n}$.

We now show that if $x$ is not in $Z(\boldsymbol{H})$ then there exists a neighborhood $U_{x}$ of $x$ in $R^{n}$ and a neighborhood $V_{x}$ of the identity in $\boldsymbol{H}$ such that, for every continuous function $f$ on $R^{n}$ and every $F$ in $C_{c}^{\infty}\left(V_{x}\right), f \nabla F$ is indefinitely differentiable in $U_{x}$. To do this, choose a diffeomorphism $\theta$ of a neighborhood of the origin $R^{m}$ onto a neighborhood $W$ of the identity in $\boldsymbol{H}$, as in the opening paragraphs of this section. If $W$ is chosen to be sufficiently small, then there exists an indefinitely differentiable function $h$ on $R^{m}$ such that

$$
\int_{H} F(A) d A=\int_{R^{m}} F(\theta(t)) h(t) d t
$$

for all $F$ in $C_{c}^{\infty}(W)$; see [3]. In particular, (3.1) becomes

$$
f \nabla F(x)=\int_{R^{m}} f(\theta(t) x) F(\theta(t)) h(t) d t
$$

for every continuous $f$ on $R^{n}$ and every $F$ in $C_{c}^{\infty}(W)$. Now, in view of the choice of $x$, there is an $i$ in $I$ such that $P_{i}(x) \neq 0$. We may, without loss of generality, assume that $i=\{1,2, \cdots, n\}$. Consider now the map

$$
\left(t_{1}, \cdots, t_{m}, y_{1}, \cdots, y_{n}\right) \longrightarrow\left(s_{1}, \cdots, s_{m}, z_{1}, \cdots, z_{n}\right)
$$

of $R^{m+n}$ into $R^{m+n}$ which is defined by

$$
\begin{aligned}
& s_{k}=\theta_{k_{1}}\left(t_{1}, \cdots, t_{m}\right) y_{1}+\cdots+\theta_{k_{n}}\left(t_{1}, \cdots, t_{m}\right) y_{n} \quad(1 \leqq k \leqq n) \\
& s_{k}=t_{k}(n+1 \leqq k \leqq m) \\
& z_{k}=y_{k}(1 \leqq k \leqq n) .
\end{aligned}
$$

It is easy to verify that the Jacobian of this transformation, evaluated at $t_{1}=\cdots=t_{m}=0, y_{1}=x_{1}, \cdots, y_{n}=x_{n}$, is just $P_{i}(x)$, which is nonzero. Thus, there exists a neighborhood $U_{x}$ of $x$ in $R^{n}$ and a neighborhood $\boldsymbol{V}_{x}$ of the identity in $\boldsymbol{H}$, such that the Jacobian of the transformation (3.3) does not vanish on $U_{x} \times \theta^{-1}\left(V_{x}\right)$. The Inverse Function theorem now tells us that the transformation, when restricted to $U_{x} \times \theta^{-1}\left(V_{x}\right)$, has an inverse

$$
\left(s_{1}, \cdots, s_{m}, z_{1}, \cdots, z_{n}\right) \longrightarrow\left(t_{1}, \cdots, t_{m}, y_{1}, \cdots, y_{n}\right)
$$

which clearly has the form

$$
\begin{array}{lrl}
t_{k} & =g_{k}\left(s_{1}, \cdots, s_{m}, z_{1}, \cdots, z_{n}\right) & (1 \leqq k \leqq m) \\
y_{k}=z_{k} & (1 \leqq k \leqq n)
\end{array}
$$


where $g_{1}, \cdots, g_{m}$, are indefinitely differentiable functions on $R^{m+n}$. We write this briefly as

$$
\begin{aligned}
& t=g(s, z) \\
& y=z .
\end{aligned}
$$

We assume that $V_{x}$ is contained in $W$. If this is the case, then, in view of (3.2),

$$
\int_{R^{n}} \phi(y) f \nabla F(y) d y=\int_{R^{n+m}} \phi(y) f(\theta(t) y) F(\theta(t)) h(t) d t d y
$$

for all $\phi$ in $C_{c}^{\infty}\left(U_{x}\right), F$ in $C_{c}^{\infty}\left(V_{x}\right)$, and each continuous function $f$ on $R^{n}$. If we now make the change of variables defined by (3.4) in the integral on the right of (3.5), we find that

$$
\begin{aligned}
\int_{R^{n}} \phi(y) f \nabla F(y) d y & =\int_{R^{n+m}} \phi(z) f^{\prime}(s) F^{\prime}(s, z) h^{\prime}(s, z) J(s, z) d s d z \\
& =\int_{R^{n+m}} \phi(y) f^{\prime}(s) F^{\prime}(s, y) h^{\prime}(s, y) J(s, y) d s d y
\end{aligned}
$$

where the functions $f^{\prime}, F^{\prime}$, and $h^{\prime}$ are defined by

$$
\begin{aligned}
f^{\prime}(s) & =f\left(s_{1}, \cdots, s_{n}\right) \\
F^{\prime}(s, z) & =F(\theta(g(s, z))) \\
h^{\prime}(s, z) & =h(g(s, z))
\end{aligned}
$$

and $J$ is the Jacobian of the transformation (3.4). From (3.6), we deduce that

$$
f \nabla F(y)=\int_{R^{m}} f^{\prime}(s) F^{\prime}(s, y) h^{\prime}(s, y) J(s, y) d s
$$

for all $y$ in $U_{x}$. Since $F^{\prime}, h^{\prime}$, and $J$ are indefinitely differentiable on $R^{m+n}$, the indefinite differentiability of $f \nabla F$ in $U_{x}$ is now evident.

We now go a step further, and show that $f \nabla F$ is indefinitely differentiable everywhere in $R^{n} \backslash Z(\boldsymbol{H})$ for all $F$ in $C_{c}^{\infty}(\boldsymbol{H})$ and all continuous functions $f$ on $R^{n}$. First, we make the observation that

$$
P_{i}(x)=\operatorname{det} A^{-1} \cdot P_{i}(A x)
$$

for all $x$ in $R^{n}, A$ in $H$, and $i$ in $I$. To see this, assume, without loss of generality, that $i=\{1,2, \cdots, n\}$. Temporarily denote by $T$ the map (3.3) of $R^{m+n}$ into itself. Then $T$ is composed of the maps

$$
(t, y) \longrightarrow\left(t, A^{-1} y\right)
$$

and

$$
(t, y) \longrightarrow T(t, A y)
$$


Hence, the Jacobian of $T$ is the product of the Jacobians of the maps (3.7) and (3.8). Evaluating these Jacobians at $t=0, y=x$, we get (3.6). It now follows that if $x$ is in $R^{n} \backslash Z(\boldsymbol{H})$ then the same is true for $A x$, for all $A$ in $\boldsymbol{H}$. Thus, in the notation of the preceding paragraph, we may choose, for each $A$ in $\boldsymbol{H}$, a neighborhood $U_{A x}$ of $A x$ and a neighborhood $V_{A x}$ of the identity in $\boldsymbol{H}$, such that $f \nabla G$ is indefinitely differentiable in $U_{A x}$ whenever $G$ is in $C_{c}^{\infty}\left(V_{A x}\right)$ and $f$ is a continuous function on $R^{n}$. Now let $F$ be an arbitrary element of $C_{c}^{\infty}(\boldsymbol{H})$. Then the collection of open sets $\left\{V_{A x} A: A \in \operatorname{supp} F\right\}$ covers the compact set $\operatorname{supp} F$ in $\boldsymbol{H}$. (Here, $\boldsymbol{V}_{A x} A$ denotes the set $\{B A: B \in$ $\left.V_{A x}\right\}$.) Thus, there exists a finite number of elements $A_{1}, \cdots, A_{r}$ in $\boldsymbol{H}$ such that

$$
\operatorname{supp} F \subset V_{A_{1} x} A_{1} \cup \cdots \cup V_{A_{r} x} A_{r} .
$$

Now choose functions $F_{1}, \cdots, F_{r}$ in $C_{c}^{\infty}(\boldsymbol{H})$ such that

$$
F_{1}+\cdots+F_{r}=1
$$

on $\operatorname{supp} F$ and

$$
\operatorname{supp} F_{k} \subset V_{A_{k} x} A_{k}
$$

for $k=1, \cdots, r$. Then we clearly have

$$
f \nabla F=f \nabla\left(F F_{1}\right)+\cdots+f \nabla\left(F F_{r}\right) .
$$

We claim that each of the functions $f \nabla\left(F F_{k}\right)$ is indefinitely differentiable on some neighborhood $U$ of $x$. To prove this, write

$$
U=A_{1}^{-1}\left(U_{A_{1} x}\right) \cap \cdots \cap A_{r}^{-1}\left(U_{A_{r} x}\right)
$$

and define (for $k=1, \cdots, r$ ) the functions $G_{k}$ by

$$
G_{k}(A)=F\left(A A_{k}\right) F_{k}\left(A A_{k}\right)
$$

Then $G_{k}$ belongs to $C_{c}^{\infty}\left(V_{A_{k} x}\right)$, and so $f \nabla G_{k}$ is indefinitely differentiable in $U_{A_{k} x}$. But, if $\Delta$ denotes the modular function of $\boldsymbol{H}$, then for each $\dot{\phi}$ in $C_{c}^{\infty}\left(R^{n}\right)$

$$
\begin{aligned}
\int_{R^{n}} f \nabla\left(F F_{k}\right)(x) \phi(-x) d x & =\int_{H}\left\langle f^{A}, \dot{\phi}\right\rangle F(A) F_{k}(A) d A \\
& =\Delta\left(A_{k}^{-1}\right) \int_{H}\left\langle f^{A A_{k}}, \dot{\phi}\right\rangle F\left(A A_{k}\right) F_{k}\left(A A_{k}\right) d A \\
& =\Delta\left(A_{k}^{-1}\right)\left|\operatorname{det} A_{k}^{-1}\right| \int_{H}\left\langle f^{A}, \phi^{A} \bar{k}^{-1}\right\rangle G_{k}(A) d A \\
& =\Delta\left(A_{k}^{-1}\right)\left|\operatorname{det} A_{k}^{-1}\right| \int_{R^{n}} f \nabla G_{k}(x) \phi^{A-1}{ }_{k}(-x) d x \\
& =\Delta\left(A_{k^{-1}}^{-1}\right) \int_{R^{n}} f \nabla G_{k}\left(A_{k} x\right) \dot{\phi}(-x) d x .
\end{aligned}
$$


Thus, we conclude that

$$
f \nabla\left(F F_{k}\right)(x)=\Delta\left(A_{k}^{-1}\right) f \nabla G_{k}\left(A_{k} x\right)
$$

for all $x$ in $R^{n}$. Since $f \nabla G_{k}$ is indefinitely differentiable in $U_{A_{k} x}$, it follows that $f \nabla\left(F F_{k}\right)$ is indefinitely differentiable in $A_{k}^{-1}\left(U_{A_{k} x}\right)$, and hence in $U$. Thus $f \nabla F$ is also indefinitely differentiable in $U$.

Now consider an arbitrary distribution $q$ in $D^{\prime}\left(R^{n}\right)$. Again, let $x$ be an arbitrary point in $R^{n} \backslash Z(\boldsymbol{H})$. We first notice that for each multi-index $\alpha$, and each $\phi$ in $C_{c}^{\infty}\left(R^{n}\right)$, we clearly have

$$
D^{\alpha}\left(\dot{\phi}^{A}\right)=\sum_{|\beta| \leqq ; \alpha \mid} G_{\beta}^{\alpha}(A)\left(D^{\beta} \dot{\phi}\right)^{A}
$$

where the $G_{\beta}^{\alpha}$ are $C^{\infty}$ functions on $\boldsymbol{H}$ depending only on $\alpha$ and $\beta$. It follows easily that for each distribution $q$ on $R^{n}$,

$$
D^{\alpha}\left(q^{A}\right)=\sum_{|\beta| \leq|\alpha|} G_{\beta}^{\alpha}(A)\left(D^{\beta} q\right)^{A}
$$

and

$$
\left(D^{\alpha} q\right)^{A}=\sum_{|\beta| \leqq|\alpha|} \check{G}_{\beta}^{\alpha}(A) D^{\beta}\left(q^{A}\right)
$$

where we have written

$$
\breve{G_{\beta}^{\alpha}}(A)=G_{\beta}^{\alpha}\left(A^{-1}\right) \quad(A \in \boldsymbol{H}) .
$$

Now choose a neighborhood $W$ of $x$ which has compact closure. Then, inside $W, q$ can be expressed as

$$
q=\sum_{\alpha} D^{\alpha} f_{\alpha}
$$

where the $f_{\alpha}$ are continuous functions on $R^{n}$, and the sum is finite. Using (3.11), we see that if $F$ is in $C_{c}^{\infty}(\boldsymbol{H})$ and $\dot{\phi}$ is in $C_{c}^{\infty}\left(R^{n}\right)$, then

$$
\begin{aligned}
\left\langle\dot{\phi},\left(D^{\alpha} f_{\alpha}\right) \nabla F\right\rangle & =\int_{H}\left\langle\left(D^{\alpha} f_{\alpha}\right)^{A}, \phi\right\rangle F(A) d A \\
& =\sum_{\beta} \int_{H}\left\langle D^{\beta}\left(f_{\alpha}^{A}\right), \dot{\phi}\right\rangle \breve{G_{\beta}^{\alpha}}(A) F(A) d A \\
& =\sum_{\beta} \int_{H}\left\langle f_{\alpha}^{A}, D^{\beta} \dot{\phi}\right\rangle \breve{G}_{\beta}^{\alpha}(A) F(A) d A \\
& =\sum_{\beta}\left\langle D^{\beta} \dot{\phi}, f_{\alpha} \nabla\left(\breve{G}_{\beta}^{\alpha} F\right)\right\rangle \\
& =\sum_{\beta}\left\langle\dot{\phi}, D^{\beta}\left[f_{\alpha} \nabla\left(\breve{G}_{\beta}^{\alpha} F\right)\right]\right\rangle .
\end{aligned}
$$

We infer that

$$
\left(D^{\alpha} f_{\alpha}\right) \nabla F=\sum_{\beta} D^{\beta}\left[f_{\alpha} \nabla\left(\breve{G_{\beta}^{\alpha}} F\right)\right]
$$


as distributions on $R^{n}$. But, in view of what we have already shown, each of the functions $f_{\alpha} \nabla\left(G_{\beta}^{\alpha} F\right)$ is indefinitely differentiable on some neighborhood $U$ of $x$. The same is therefore true of the distributions $\left(D^{\alpha} f_{\alpha}\right) \nabla F$. Now, it is evident that

$$
q \nabla F=\sum_{\alpha}\left(D^{\alpha} f_{\alpha}\right) \nabla F
$$

in $W$. Since we may obviously assume that $U$ is contained in $W$, it now follows that the distribution $q \nabla F$ is an indefinitely differentiable function in the neighborhood $U$ of $x$. This completes the proof of the first assertion in Lemma 3.1.

To prove the second assertion, we refer to (3.10) and carry out a computation similar to (3.12).

Lemma 3.2. Suppose that $\boldsymbol{H}$ has dimension $m \geqq n$. Let $q$ be a distribution on $R^{n}$ and $x$ a point in supp $q$ which is not in $Z(\boldsymbol{H})$. Then there is a function $F$ in $C_{c}^{\infty}(\boldsymbol{H})$ such that $q \nabla F(x) \neq 0$.

Proof. Write $S$ for the set of all points $z$ in $R^{n} \backslash Z(H)$ such that, for some $F$ in $C_{c}^{\infty}(H), q \nabla F(z) \neq 0$.

We first observe that $x$ is in the closure of $S$. For suppose that there were a neighborhood $U$ of $x$ which contained no points of $S$. This would entail that each function $q \nabla F$ vanishes identically in $U$. It would then follow that, for each $\phi$ in $C_{c}^{\infty}(U)$ and each $F$ in $C_{c}^{\infty}(\boldsymbol{H})$,

$$
\begin{aligned}
\int_{G}\left\langle q^{A}, \dot{\phi}\right\rangle F(A) d A & =\int_{R^{n}} q \nabla F(y) \dot{\phi}(-y) d y \\
& =0 .
\end{aligned}
$$

This would lead to the conclusion that the function $A \rightarrow\left\langle q^{A}, \phi\right\rangle$ is identically zero on $\boldsymbol{H}$, for every $\phi$ in $C_{c}^{\infty}(U)$. In particular, we would have

$$
\langle q, \phi\rangle=0 \quad\left(\phi \in C_{c}^{\infty}(U)\right)
$$

contradicting the fact that $x$ is in $\operatorname{supp} q$.

We know that $P_{i}(x) \neq 0$ for some $i$, and, as usual, we assume that $i=\{1,2, \cdots, n\}$. Consider the map

$$
\left(t_{1}, \cdots, t_{m}\right) \longrightarrow\left(y_{1}, \cdots, y_{m}\right)
$$

of $R^{m}$ into itself defined by

$$
\begin{array}{lr}
y_{k}=\theta_{k 1}\left(t_{1}, \cdots, t_{m}\right) x_{1}+\cdots+\theta_{k n}\left(t_{1}, \cdots, t_{m}\right) x_{n} & (1 \leqq k \leqq n) \\
y_{k}=t_{k} & (n+1 \leqq k \leqq m) .
\end{array}
$$

The Jacobian of this transformation is $P_{i}(x)$, and is therefore nonzero at $t_{1}=\cdots=t_{m}=0$. Hence this map carries a neighborhood $W$ of 
the origin in $R^{m}$ onto a neighborhood of the point $\left(x_{1}, \cdots, x_{n}, 0, \cdots, 0\right)$. It follows that the set $\{\theta(t) x: t \in W\}$ is a neighborhood of $x$ in $R^{n}$ and, consequently, so is the set $\{B x: B \in \mathbb{H}\}$. Since $x$ is in the closure of $S$, there must be a point $z$ in $S$ which has the form $z=B x$, for some $B$ in $\boldsymbol{H}$. Choose a function $G$ in $C_{c}^{\infty}(\boldsymbol{H})$ such that $q \nabla G(z) \neq 0$, and define the function $F$ by

$$
F(A)=G\left(A B^{-1}\right)
$$

Then $F$ is in $C_{c}^{\infty}(\boldsymbol{H})$. Moreover, using an argument similar to one used in the course of the proof of Lemma 3.1, it may be shown that

$$
q \nabla F(y)=q \nabla G(B y)
$$

It follows that

$$
q \nabla F(x)=q \nabla G(z) \neq 0
$$

as we wished to show.

4. A cancellation theorem. In this section, we prove our main theorem. Before we do so, however, it is necessary to make some brief comments about notation. We shall want to make use of the Schwartz-Fourier transform. Now, if $q$ is a temperate distribution and $A$ is in $\mathrm{Gl}(n, R)$, then it is easy to verify that

$$
\left(q^{A}\right)^{\wedge}=\left|\operatorname{det} A^{-1}\right| \hat{q}^{A^{\prime-1}} \text {. }
$$

It appears, then, that we shall be dealing with both a closed subgroup $G$ of $G l(n, R)$ and its adjoint group $G^{\prime}$. It is actually to the group $G^{\prime}$ that we shall apply the theory developed in the preceding section. As far as the operation $\nabla$ is concerned, we shall use it in both the case when we are considering the group $G$ and in the case when we are considering the group $G^{\prime}$. Thus, if $q$ is a distribution on $R^{n}$ and $F$ is in $C_{c}^{\infty}(G)$, we shall write $q \nabla F$ for the distribution defined in $\S 3$ (with $\boldsymbol{G}$ playing the role of $\boldsymbol{H}$ ); but also, if $q$ is a distribution and $F^{\prime}$ is in $C_{c}^{\infty}\left(G^{\prime}\right)$, we shall write $q \nabla F^{\prime}$ for the distribution arising when we replace $\boldsymbol{H}$ by $G^{\prime}$ in the definitions of $\S 3$. The context makes evident the group ( $G$ or $G^{\prime}$ ) with respect to which the operation $\nabla$ is being defined.

We begin with a lemma. This will then yield the main theorem as an easy corollary.

Lemma 4.1. Let $G$ be a closed subgroup of $\mathrm{Gl}(n, R)$ of dimension $m \geqq n$. Let $E$ be a barrelled $G$-space. Then for all $u$ in $E, v$ in $E^{\prime}, F^{\prime}$ in $C_{c}^{\infty}\left(G^{\prime}\right)$, and $\rho$ in $C_{c}^{\infty}\left(R^{n}\right)$ such that 


$$
\operatorname{supp} \phi \subset R^{n} \backslash Z\left(G^{\prime}\right)
$$

we have the identity

$$
\left\langle\phi, \widehat{u} \nabla F^{\prime} \cdot \hat{v}\right\rangle=\int_{G^{\prime}}\left\langle u^{A^{-1}}, v * \hat{\phi}\right\rangle F^{\prime}\left(A^{\prime}\right) d A^{\prime} .
$$

Proof. Recall that if $q$ is a temperate distribution, then so is $q \nabla F^{\prime}$. Thus if $\phi$ is in $C_{c}^{\infty}\left(R^{n}\right)$, then

$$
\begin{aligned}
{\left[q \nabla F^{\prime} \cdot \phi\right]^{\wedge}(x) } & =\left(q \nabla F^{\prime}\right)^{\wedge} * \hat{\phi}(-x) \\
& =\left\langle\hat{\phi}_{x},\left(q \nabla F^{\prime}\right)^{\wedge}\right\rangle \\
& =\left\langle\left(\hat{\phi}_{x}\right)^{\wedge}, q \nabla F^{\prime}\right\rangle \\
& =\int_{G^{\prime}}\left\langle q^{A^{\prime}},\left(\hat{\phi}_{x}\right)^{\wedge}\right\rangle F^{\prime}\left(A^{\prime}\right) d A^{\prime} \\
& =\int_{G^{\prime}}\left\langle\left(q^{A^{\prime}}\right)^{\wedge}, \hat{\phi}_{x}\right\rangle F^{\prime}\left(A^{\prime}\right) d A^{\prime} \\
& =\int_{G^{\prime}}\left|\operatorname{det} A^{-1}\right|\left\langle q^{A^{-1}}, \phi_{x}\right\rangle F^{\prime}\left(A^{\prime}\right) d A^{\prime} \\
& =\int_{G^{\prime}}\left|\operatorname{det} A^{-1}\right| q^{A^{-1}} * \phi(x) F^{\prime}\left(A^{\prime}\right) d A^{\prime}
\end{aligned}
$$

Now consider a temperate distribution $v$, a function $F^{\prime}$ in $C_{c}^{\infty}\left(G^{\prime}\right)$, and functions $\psi$ in $S\left(R^{n}\right)$ and $\phi$ in $C_{c}^{\infty}\left(R^{n}\right)$. Then, if $\left(\eta_{i}\right)$ is a net extracted from $S\left(R^{n}\right)$ which converges to $v$ in $S^{\prime}\left(R^{n}\right)$, we have, by virtue of (4.2),

$$
\begin{aligned}
\left\langle\phi, \hat{\psi} \nabla F^{\prime} \cdot \hat{v}\right\rangle & =\lim _{i}\left\langle\hat{\psi} \nabla F^{\prime} \cdot \phi, \hat{\eta}_{i}\right\rangle \\
& =\lim _{i} \int_{R^{n}}\left[\hat{\psi} \nabla F^{\prime} \cdot \phi\right]^{\wedge}(x) \eta_{i}(-x) d x \\
& =\lim _{i} \int_{R^{n}} \int_{G^{\prime}}\left|\operatorname{det} A^{-1}\right| \psi^{A^{-1}} * \hat{\phi}(x) F^{\prime}\left(A^{\prime}\right) d A^{\prime} \eta_{i}(-x) d x \\
& =\lim _{i} \int_{G^{\prime}}\left|\operatorname{det} A^{-1}\right| \psi^{A^{-1}} * \hat{\phi} * \eta_{i}(0) F^{\prime}\left(A^{\prime}\right) d A^{\prime} .
\end{aligned}
$$

Now, the set

$$
\left\{\psi^{A^{-1}} * \hat{\phi}: A^{\prime} \in \operatorname{supp} F^{\prime}\right\}
$$

is a bounded subset of $S\left(R^{n}\right)$. Hence

$$
\lim _{i} \psi^{A^{-1}} * \hat{\phi} * \eta_{i}(0)=\psi^{A^{-1}} * \hat{\phi} * v(0)
$$

uniformly for $A^{\prime}$ in supp $F^{\prime}$. Thus (4.3) becomes

$$
\left\langle\phi, \hat{\psi} \nabla F^{\prime} \cdot \hat{v}\right\rangle=\int_{G^{\prime}}\left|\operatorname{det} A^{-1}\right| \psi^{A^{-1}} * \hat{\phi} * v(0) F^{\prime}\left(A^{\prime}\right) d A^{\prime} .
$$

Now consider a fixed $\phi$ in $C_{c}^{\infty}\left(R^{n}\right)$ with 


\section{$\operatorname{supp} \phi \subset R^{n} \backslash Z\left(G^{\prime}\right)$}

and a fixed $F^{\prime \prime}$ in $C_{c}^{\infty}\left(G^{\prime}\right)$. Then, for each $u$ in $E, \hat{u} \nabla F^{\prime}$ is indefinitely differentiable on a neighborhood of $\operatorname{supp} \phi$ (by Lemma 3.1). Thus $\hat{u} \nabla F^{\prime} \cdot \phi$ is in $C_{c}^{\infty}\left(R^{n}\right)$ and so $\left[u \nabla F^{\prime} \cdot \phi\right]^{\wedge}$ is in $S\left(R^{n}\right)$. We claim that the map

$$
u \longrightarrow\left[\hat{u} \nabla F^{\prime} \cdot \phi\right]^{\wedge}
$$

is continuous from $E$ into $S\left(R^{n}\right)$. Since $E$ is barrelled and $S\left(R^{n}\right)$ is fully complete, it suffices to show that the graph of this map is closed. Thus, assume that $\left(u_{i}\right)$ converges to $u$ in $E$ and that

$$
\lim _{i}\left[\widehat{u}_{i} \nabla F^{\prime} \cdot \phi\right]^{\wedge}=\eta
$$

in $S\left(R^{n}\right)$. Then, for each $\lambda$ in $C_{c}^{\infty}\left(R^{n}\right)$, we have, in view of (4.2),

$$
\begin{aligned}
\eta * \lambda(0) & =\lim _{i}\left[\hat{u}_{i} \nabla F^{\prime} \cdot \phi\right]^{\wedge} * \lambda(0) \\
& =\lim _{i} \int_{R^{n}}\left[\hat{u}_{i} \nabla F^{\prime} \cdot \phi\right]^{\wedge}(x) \lambda(-x) d x \\
& =\lim _{i} \int_{R^{n}} \int_{G^{\prime}}\left|\operatorname{det} A^{-1}\right| u_{i}^{A^{-1}} * \hat{\phi}(x) F^{\prime}\left(A^{\prime}\right) \lambda(-x) d A^{\prime} d x \\
& =\lim _{i} \int_{G^{\prime}}\left|\operatorname{det} A^{-1}\right| u_{i}^{A-1} * \hat{\phi} * \lambda(0) F^{\prime}\left(A^{\prime}\right) d A^{\prime} \\
& =\lim _{i} \int_{G^{\prime}}\left\langle u_{i},(\hat{\phi} * \lambda)^{A}\right\rangle F^{\prime}\left(A^{\prime}\right) d A^{\prime} .
\end{aligned}
$$

Now, since $\operatorname{supp} F^{\prime}$ is compact, the set $\left\{A: A^{\prime} \in \operatorname{Supp} F^{\prime}\right\}$ is a compact subset of $G$. Hence, since $E$ is a $G$-space, the set

$$
\left\{(\hat{\phi} * \lambda)^{A}: A^{\prime} \in \operatorname{supp} F^{\prime}\right\}
$$

is a weakly compact, and hence equicontinuous ( $E$ being barrelled) subset of $E^{\prime}$. It follows that

$$
\lim _{i}\left\langle u_{i},(\hat{\phi} * \lambda)^{A}\right\rangle=\left\langle u,(\hat{\phi} * \lambda)^{A}\right\rangle
$$

uniformly for $A^{\prime}$ in supp $F^{\prime}$. Using this in (4.6), we get

$$
\begin{aligned}
\eta * \lambda(0) & =\int_{G^{\prime}}\left\langle u,(\hat{\phi} * \lambda)^{A}\right\rangle F^{\prime}\left(A^{\prime}\right) d A^{\prime} \\
& =\int_{G^{\prime}}\left|\operatorname{det} A^{-1}\right|\left\langle u^{A^{-1}}, \hat{\phi} * \lambda\right\rangle F^{\prime}\left(A^{\prime}\right) d A^{\prime} \\
& =\int_{G^{\prime}}\left|\operatorname{det} A^{-1}\right| u^{A^{-1}} * \hat{\phi} * \lambda(0) F^{\prime}\left(A^{\prime}\right) d A^{\prime} \\
& =\int_{R^{n}} \int_{G^{\prime}}\left|\operatorname{det} A^{-1}\right| u A^{-1} * \hat{\phi}(x) F^{\prime \prime}\left(A^{\prime}\right) d A^{\prime} \lambda(-x) d x \\
& =\frac{\hat{u} \nabla F^{\prime} \cdot \phi * \lambda(0) .}{}
\end{aligned}
$$


We conclude that $\eta$ coincides with $\left[\hat{u} \nabla F^{\prime \prime} \cdot \phi\right]^{\wedge}$ and therefore the graph of the map (4.5) is indeed closed.

Now consider again a function $\phi$ in $C_{c}^{\infty}\left(R^{n}\right)$ with

$$
\operatorname{supp} \phi \subset R^{n} \backslash Z\left(G^{\prime}\right) \text {. }
$$

Let $\left(\psi_{i}\right)$ be a net extracted from $S\left(R^{n}\right)$ which converges to $u$ in $E$. Using the continuity of the map (4.5) and relation (4.4), we find that

$$
\begin{aligned}
\left\langle\phi, \hat{u} \nabla F^{\prime} \cdot \hat{v}\right\rangle & =\left\langle\hat{u} \nabla F^{\prime} \cdot \phi, \hat{v}\right\rangle \\
& =\lim _{i}\left\langle\hat{\psi}_{i} \nabla F^{\prime} \cdot \phi, \hat{v}\right\rangle \\
& =\lim _{i} \int_{G^{\prime}}\left|\operatorname{det} A^{-1}\right| \psi_{i}^{A^{-1}} * \hat{\phi} * v(0) F^{\prime}\left(A^{\prime}\right) d A^{\prime} \\
& =\lim _{i} \int_{G^{\prime}}\left|\operatorname{det} A^{-1}\right|\left\langle\psi_{i}^{A}-1, \hat{\phi} * v\right\rangle F^{\prime \prime}\left(A^{\prime}\right) d A^{\prime} \\
& =\int_{G^{\prime}}\left|\operatorname{det} A^{-1}\right|\left\langle u^{A-1}, \hat{\phi} * v\right\rangle F^{\prime}\left(A^{\prime}\right) d A^{\prime}
\end{aligned}
$$

the last equality following from a now familiar argument. This completes the proof of our lemma.

THEOREM 4.2. Let $\boldsymbol{G}$ be a closed subgroup of $\mathrm{Gl}(n, R)$ of dimension $m \geqq n$. Suppose that $E$ is a barrelled $G$-space. Let $u$ in $E$ and $v$ in $E^{\prime}$ be such that

$$
\left\langle u_{b}^{A}, v\right\rangle=0
$$

for all $A$ in $G$ and $b$ in $R^{n}$. Then

$$
\operatorname{supp} \hat{v} \cap\left\{\bigcup_{A \in G} A^{\prime}(\operatorname{supp} \hat{u})\right\} \subset Z\left(G^{\prime}\right) \text {. }
$$

Proof. Relation (4.7) entails that

$$
\left\langle u^{A}, \psi * v\right\rangle=0
$$

for all $\psi$ in $S\left(R^{n}\right)$. Lemma 4.1 now tells us that $\hat{u} \nabla F^{\prime} \cdot \hat{v}$ vanishes in $R^{n} \backslash Z\left(G^{\prime}\right)$ for all $F^{\prime}$ in $C_{c}^{\infty}\left(G^{\prime}\right)$. Lemma 3.2 now leads us to the conclusion that

$$
\operatorname{supp} \hat{v} \cap \operatorname{supp} \widehat{u}^{A^{\prime}-1} \subset Z\left(G^{\prime}\right)
$$

for each $A$ in $\boldsymbol{G}$. Since

$$
\operatorname{supp} \widehat{u}^{A^{\prime-1}}=A^{\prime}(\operatorname{supp} \hat{u})
$$

the desired result follows.

5. A density theorem. Let $E$ be a subspace of $S^{\prime}\left(R^{n}\right)$. If $S$ 
is a subset of $R^{n}$, we say that $S$ is a set of uniqueness for $E$ relations

$$
\operatorname{supp} \hat{v} \subset S, v \in E
$$

entail that $v=0$.

TheORem 5.1. Let $\boldsymbol{G}$ be a closed subgroup of $\mathrm{Gl}(n, R)$ of dimension $m \geqq n$. Suppose that $E$ is a barrelled $G$-space and that the set $Z\left(G^{\prime}\right)$ is a set of uniqueness for $E^{\prime}$. Let $u$ be an element of $E$. Then $T_{G}[u]$ coincides with the whole of $E$ if and only if

$$
\mathbf{U}\left\{A^{\prime}(\operatorname{supp} u): A \in \boldsymbol{G}\right\}
$$

is dense in $R^{n}$.

Proof. The density of the set (5.1) is clearly necessary for $T_{c}$ [ $u$ ] to coincide with $E$. For suppose that some nonvoid open set $U$ in $R^{n}$ did not intersect the set (5.1). Consider a nonzero $\phi$ in $C_{c}^{\infty}\left(R^{n}\right)$ with support in $U$. Then its Fourier transform $\hat{\phi}$ is a nonzero element of $E^{\prime}$ which annihilates $T_{G}[u]$.

To prove the converse, suppose that (5.1) is dense. Let $x$ be in $R^{n} \backslash Z\left(G^{\prime}\right)$. Then, as in the proof of Lemma 3.2, we deduce that $\left\{A^{\prime} x: A \in G\right\}$ is a neighborhood of $x$. Thus, there exists a point in the set (5.1) which is also in $\left\{A^{\prime} x: A \in G\right\}$. In other words, there is a point $z$ in supp $\hat{u}$, and elements $A, B$ in $G$, such that $A^{\prime} z=B^{\prime} x$. Hence, $x=B^{\prime-1} A^{\prime} z$ is in the set (5.1). By Theorem 4.2, $x$ cannot belong to the support of the Fourier transform of any distribution $v$ in $E^{\prime}$ which annihilates $T_{G}[u]$. It follows that if $v$ annibilates $T_{G}[u]$, then we must have supp $\hat{v}$ contained in $Z\left(G^{\prime}\right)$. Since $Z\left(G^{\prime}\right)$ is a set of uniqueness for $E^{\prime}$ by assumption, we infer that $v=0$. The Hahn-Banach theorem now tells us that $T_{G}[u]$ is the whole of $E$.

The problem considered above has been studied by Gosselin [1] in the case when $E$ is the space Lebesgue square integrable functions, but with no restriction on the dimension of $G$. The approach in [1] is a measure theoretic one.

\section{REFERENCES}

1. R. P. Gosselin, Closure theorems for affine transformation groups, (To appear).

2. S. R. Harasymiv, On approximation by dilations of distributions, Pacific J. Math., (1969), 463-474.

3. Frank W. Warner, Foundations of Differentiable Manifolds and Lie Groups, Scott, Foreman and Company, 1971.

4. K. Yoshinaga and H. Ogata, On convolutions, J. Sci. Hiroshima Univ., Ser. A, 22 (1958), 15-24.

Received June 6, 1973 and in revised form January 24, 1974. 



\section{PACIFIC JOURNAL OF MATHEMATICS}

\section{EDITORS}

RICHARD ARENS (Managing Editor)

University of California

Los Angeles, California 90024

\section{R. A. Beaumont \\ University of Washington \\ Seattle, Washington 98105}

\section{J. DugundJI}

Department of Mathematics

University of Southern California

Los Angeles, California 90007

D. Gilbarg and J. Milgram

Stanford University

Stanford, California 94305

\section{ASSOCIATE EDITORS}
E. F. BECKENBACH
B. H. NeumanN
F. WOLF
K. YOSHIDA

\section{SUPPORTING INSTITUTIONS}

UNIVERSITY OF BRITISH COLUMBIA
CALIFORNIA INSTITUTE OF TECHNOLOGY
UNIVERSITY OF CALIFORNIA
MONTANA STATE UNIVERSITY
UNIVERSITY OF NEVADA
NEW MEXICO STATE UNIVERSITY
OREGON STATE UNIVERSITY
UNIVERSITY OF OREGON
OSAKA UNIVERSITY

UNIVERSITY OF BRITISH COLUMBIA

UNIVERSITY OF CALIFORNIA

MONTANA STATE UNIVERSITY

NEW MEXICO STATE UNIVERSITY

OREGON STATE UNIVERSITY

OSAKA UNIVERSITY

\author{
UNIVERSITY OF SOUTHERN CALIFORNIA \\ STANFORD UNIVERSITY \\ UNIVERSITY OF TOKYO \\ UNIVERSITY OF UTAH \\ WASHINGTON STATE UNIVERSITY \\ UNIVERSITY OF WASHINGTON \\ AMERICAN MATHEMATICAL SOCIETY \\ NAVAL WEAPONS CENTER
}

The Supporting Institutions listed above contribute to the cost of publication of this Journal, but they are not owners or publishers and have no responsibility for its content or policies.

Mathematical papers intended for publication in the Pacific Journal of Mathematics should be in typed form or offset-reproduced, (not dittoed), double spaced with large margins. Underline Greek letters in red, German in green, and script in blue. The first paragraph or two must be capable of being used separately as a synopsis of the entire paper. Items of the bibliography should not be cited there unless absolutely necessary, in which case they must be identified by author and Journal, rather than by item number. Manuscripts, in triplicate, may be sent to any one of the editors. Please classify according to the scheme of Math. Reviews, Index to Vol. 39. All other communications should be addressed to the managing editor, or Elaine Barth, University of California, Los Angeles, California, 90024.

The Pacific Journal of Mathematics expects the author's institution to pay page charges, and reserves the right to delay publication for nonpayment of charges in case of financial emergency.

100 reprints are provided free for each article, only if page charges have been substantially paid. Additional copies may be obtained at cost in multiples of 50 .

The Pacific Journal of Mathematics is issued monthly as of January 1966. Regular subscription rate: $\$ 72.00$ a year (6 Vols., 12 issues). Special rate: $\$ 36.00$ a year to individual members of supporting institutions.

Subscriptions, orders for back numbers, and changes of address should be sent to Pacific Journal of Mathematics, 103 Highland Boulevard, Berkeley, California, 94708.

PUBLISHED BY PACIFIC JOURNAL OF MATHEMATICS, A NON-PROFIT CORPORATION

Printed at Kokusai Bunken Insatsusha (International Academic Printing Co., Ltd.), 270, 3-chome Totsuka-cho, Shinjuku-ku, Tokyo 160, Japan.

Copyright (C) 1973 by Pacific Journal of Mathematics Manufactured and first issued in Japan 


\section{Pacific Journal of Mathematics \\ Vol. 55, No. $2 \quad$ October, 1974}

Walter Allegretto, On the equivalence of two types of oscillation for elliptic

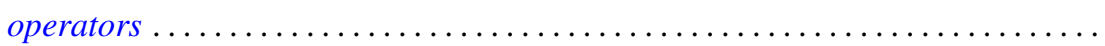

Edward Arthur Bertram, A density theorem on the number of conjugacy classes in

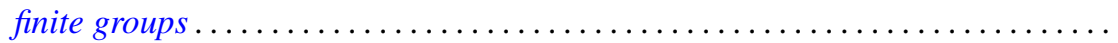

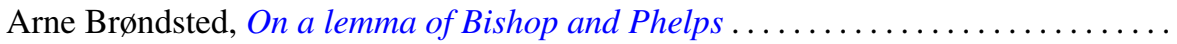

Jacob Burbea, Total positivity and reproducing kernels ..................

Ed Dubinsky, Linear Pincherle sequences . . . . . . . . . . . . . . . . . .

Benny Dan Evans, Cyclic amalgamations of residually finite groups .............

361

Barry J. Gardner and Patrick Noble Stewart, A "going down" theorem for certain

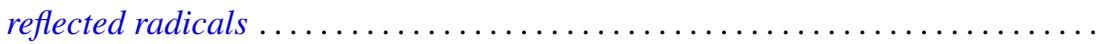

381

Jonathan Light Gross and Thomas William Tucker, Quotients of complete graphs:

revisiting the Heawood map-coloring problem ....................

Sav Roman Harasymiv, Groups of matrices acting on distribution spaces .........

Robert Winship Heath and David John Lutzer, Dugundji extension theorems for

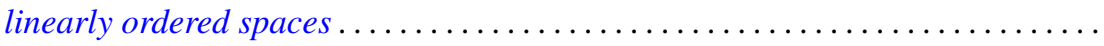

Chung-Wu Ho, Deforming p. l. homeomorphisms on a convex polygonal

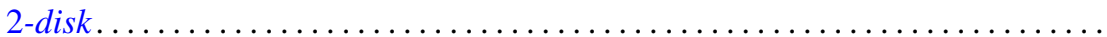

Richard Earl Hodel, Metrizability of topological spaces .................

Wilfried Imrich and Mark E. Watkins, On graphical regular representations of

cyclic extensions of groups .......................... 461

Jozef Krasinkiewicz, Remark on mappings not raising dimension of curves ..... . 479

Melven Robert Krom, Infinite games and special Baire space extensions . . . . . . 483

S. Leela, Stability of measure differential equations . . . . . . . . . . . . . . . . 489

M. H. Lim, Linear transformations on symmetric spaces . . . . . . . . . . . . . . . 499

Teng-Sun Liu, Arnoud C. M. van Rooij and Ju-Kwei Wang, On some group algebra modules related to Wiener's algebra $M_{1} \ldots \ldots \ldots \ldots \ldots \ldots \ldots \ldots \ldots \ldots \ldots$

Dale Wayne Myers, The back-and-forth isomorphism construction ............ 521

Donovan Harold Van Osdol, Extensions of sheaves of commutative algebras by

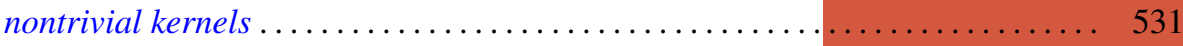

Alan Rahilly, Generalized Hall planes of even order ................... 543

Joylyn Newberry Reed, On completeness and semicompleteness of first countable

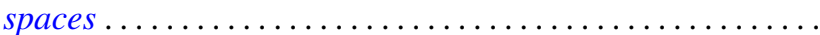

Alan Schwartz, Generalized convolutions and positive definite functions associated

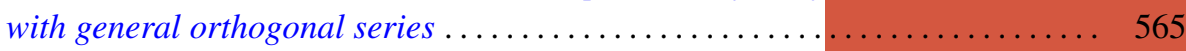

Thomas Jerome Scott, Monotonic permutations of chains . . . . . . . . . . . 583

Eivind Stensholt, An application of Steinberg's construction of twisted groups .... 595

Yasuji Takeuchi, On strongly radicial extensions . . . . ................. 619

William P. Ziemer, Some remarks on harmonic measure in space . . . . . . . . . . 629

John Grant, Corrections to: “Automorphisms definable by formulas” . . . . . . . . 639

Peter Michael Rosenthal, Corrections to: "On an inversion for the general

Mehler-Fock transform pair" ......................... 640

Carl Clifton Faith, Corrections to: "When are proper cyclics injective” . . . . . . 640 